hep-ph/0611372

\title{
Electroweak Precision Data and Gravitino Dark Matter*
}

\author{
S. HEINEMEYER ${ }^{1 \dagger}$ \\ ${ }^{1}$ Instituto de Fisica de Cantabria (CSIC-UC), Santander, Spain
}

\begin{abstract}
Electroweak precision measurements can provide indirect information about the possible scale of supersymmetry already at the present level of accuracy. We review present day sensitivities of precision data in mSUGRA-type models with the gravitino as the lightest supersymmetric particle (LSP). The $\chi^{2}$ fit is based on $M_{W}, \sin ^{2} \theta_{\text {eff }},(g-2)_{\mu}, \operatorname{BR}(b \rightarrow s \gamma)$ and the lightest MSSM Higgs boson mass, $M_{h}$. We find indications for relatively light soft supersymmetry-breaking masses, offering good prospects for the LHC and the ILC, and in some cases also for the Tevatron.
\end{abstract}

\footnotetext{
*talk given at the LCWSO6, 9-13 March 2006, Bangalore, India

${ }^{\dagger}$ email: Sven.Heinemeyer@cern.ch
} 
PRAMANA

- journal of

physics (c) Indian Academy of Sciences

pp. $1-4$

\title{
Electroweak Precision Data and Gravitino Dark Matter
}

\author{
S. Heinemeyer ${ }^{1}$ \\ ${ }^{1}$ Instituto de Fisica de Cantabria (CSIC-UC), Santander, Spain
}

\begin{abstract}
Electroweak precision measurements can provide indirect information about the possible scale of supersymmetry already at the present level of accuracy. We review present day sensitivities of precision data in mSUGRA-type models with the gravitino as the lightest supersymmetric particle (LSP). The $\chi^{2}$ fit is based on $M_{W}, \sin ^{2} \theta_{\text {eff }},(g-2)_{\mu}, \mathrm{BR}(b \rightarrow s \gamma)$ and the lightest MSSM Higgs boson mass, $M_{h}$. We find indications for relatively light soft supersymmetry-breaking masses, offering good prospects for the LHC and the ILC, and in some cases also for the Tevatron.
\end{abstract}

Keywords. gravitino dark matter, fit, precision observables

PACS Nos 2.0

\section{Introduction}

We have recently analyzed $[1,2]$ the indications provided by current experimental data concerning the possible scale of supersymmetry (SUSY) within the framework of the minimal supersymmetric extension of the Standard Model (MSSM), assuming that the soft supersymmetry-breaking scalar masses $m_{0}$, gaugino masses $m_{1 / 2}$ and tri-linear parameters $A_{0}$ were each constrained to be universal at the input GUT scale, with the gravitino heavy and the lightest supersymmetric particle (LSP) being the lightest neutralino $\tilde{\chi}_{1}^{0}$ (CMSSM). (For other recent analyses, see Ref. [3].) However, there are more scenarios for SUSY phenomenology. As an example, the gravitino might be the LSP and constitute the dark matter [4] (see also Ref. [5]), a framework known as the GDM [6].

Supersymmetry may provide an important contribution to loop effects that are rare or forbidden within the Standard Model. Especially sensitive in this respect are the observables $M_{W}$ and $\sin ^{2} \theta_{\text {eff }}$, the loop induced quantities $(g-2)_{\mu}$ and $\operatorname{BR}(b \rightarrow s \gamma)$, as well as and the lightest MSSM Higgs boson mass, $M_{h}$, (see Ref. [7] for a review). Another important constraint is provided by the cold dark matter (CDM) density $\Omega_{\mathrm{CDM}} h^{2}$ determined by WMAP and other observations. We analyze the precision observables in the context of the GDM, focusing on parameter combinations that fulfill $0.094<\Omega_{\mathrm{CDM}} h^{2}<0.129$ [8]. In order to simplify the analysis in a motivated manner, we furthermore restrict our attention to scenarios inspired by supergravity (mSUGRA), in which the gravitino mass is constrained to equal $m_{0}$ at the input GUT scale, and the trilinear and bilinear soft supersymmetry-breaking parameters are related by $A_{0}=B_{0}+m_{0}$. In the cases we review here, namely $A_{0} / m_{0}=0,3 / 4,3-\sqrt{3}, 2$, the regions of the $\left(m_{1 / 2}, m_{0}\right)$ plane allowed by cosmological constraints then take the form of wedges located at small values of $m_{0}[6,9]$. 


\section{The $\chi^{2}$ fit}

In this Section we review briefly the experimental data set that has been used for the fits. We focus on parameter points that yield the correct value of the cold dark matter density, $0.094<\Omega_{\mathrm{CDM}} h^{2}<0.129$ [8], which is, however, not included in the fit itself. The top quark mass has been fixed to $m_{t}=172.7 \mathrm{GeV}$ [10], where the experimental uncertainty of $\delta m_{t}^{\exp }=2.9 \mathrm{GeV}$ has been taken into account in the parametric uncertainty, see below. For the other observables we use the following experimental values (see Ref. [2] and references therein)

$$
\begin{aligned}
M_{W}^{\exp } & =80.410 \pm 0.032 \mathrm{GeV}, \\
\sin ^{2} \theta_{\mathrm{eff}}^{\exp } & =0.23153 \pm 0.00016, \\
a_{\mu}^{\exp }-a_{\mu}^{\text {theo,SM }} & =(25.2 \pm 9.2) \times 10^{-10} \\
\mathrm{BR}(b \rightarrow s \gamma) & =\left(3.39_{-0.27}^{+0.30}\right) \times 10^{-4}
\end{aligned}
$$

An update to the most recent experimental values would not change our results in a qualitative manner (see e.g. Ref. [11] for an analysis of the dependence on $m_{t}^{\exp }$ and $\delta m_{t}^{\exp }$ ). For $M_{h}$ we use the complete likelihood information available from LEP [12]. Our starting points are the $C L_{s}\left(M_{h}\right)$ values provided by the final LEP results on the SM Higgs boson search, see Fig. 9 in [12], where the $\chi^{2}$ contribution is obtained by inversion $C L_{s}\left(M_{h}\right)$, see Ref. [2] for details.

Assuming that the five observables listed above are uncorrelated, a $\chi^{2}$ fit has been performed with

$$
\chi^{2} \equiv \sum_{n=1}^{4}\left(\frac{R_{n}^{\exp }-R_{n}^{\text {theo }}}{\sigma_{n}}\right)^{2}+\chi_{M_{h}}^{2} .
$$

Here $R_{n}^{\exp }$ denotes the experimental central value of the $n$th observable $\left(M_{W}, \sin ^{2} \theta_{\text {eff }}\right.$, $(g-2)_{\mu}$ and $\left.\operatorname{BR}(b \rightarrow s \gamma)\right), R_{n}^{\text {theo }}$ is the corresponding GDM prediction and $\sigma_{n}$ denotes the combined error (experimental, parametric, intrinsic, Refs. [2,7]). $\chi_{M_{h}}^{2}$ denotes the $\chi^{2}$ contribution coming from the lightest MSSM Higgs boson mass as described above. For details of the theory evaluations see Refs. [7,13-16] (and references therein).

\section{Results in the GDM}

In Fig. 1 we show the total $\chi^{2}$ as a function of $m_{1 / 2}$ and various SUSY particle masses. The global minimum of $\chi^{2}$ for all the GDM models with $A_{0} / m_{0}=0,0.75,3-\sqrt{3}$ and 2 is at $m_{1 / 2} \sim 450 \mathrm{GeV}$. However, this minimum is not attained for GDM models with larger $m_{0}$, as they do not reach the low- $m_{1 / 2}$ tip of the GDM wedge. In general, we see in the different panels of Fig. 1 that there might be some hope to observe the lightest $\tilde{\tau}$ at the Tevatron, that there are good prospects for observing the $\tilde{g}$ and perhaps the $\tilde{t}_{1}$ at the LHC, and that the ILC(500) has good prospects for the $\tilde{\chi}_{1}^{0}$ and $\tilde{\tau}_{1}$, though these diminish for larger $m_{0}$. The ILC(1000) offers much better chances also for large $m_{0}$. We recall that, in these GDM scenarios, the $\tilde{\tau}_{1}$ is the NLSP, and that the $\tilde{\chi}_{1}^{0}$ is heavier. The $\tilde{\tau}_{1}$ decays into 

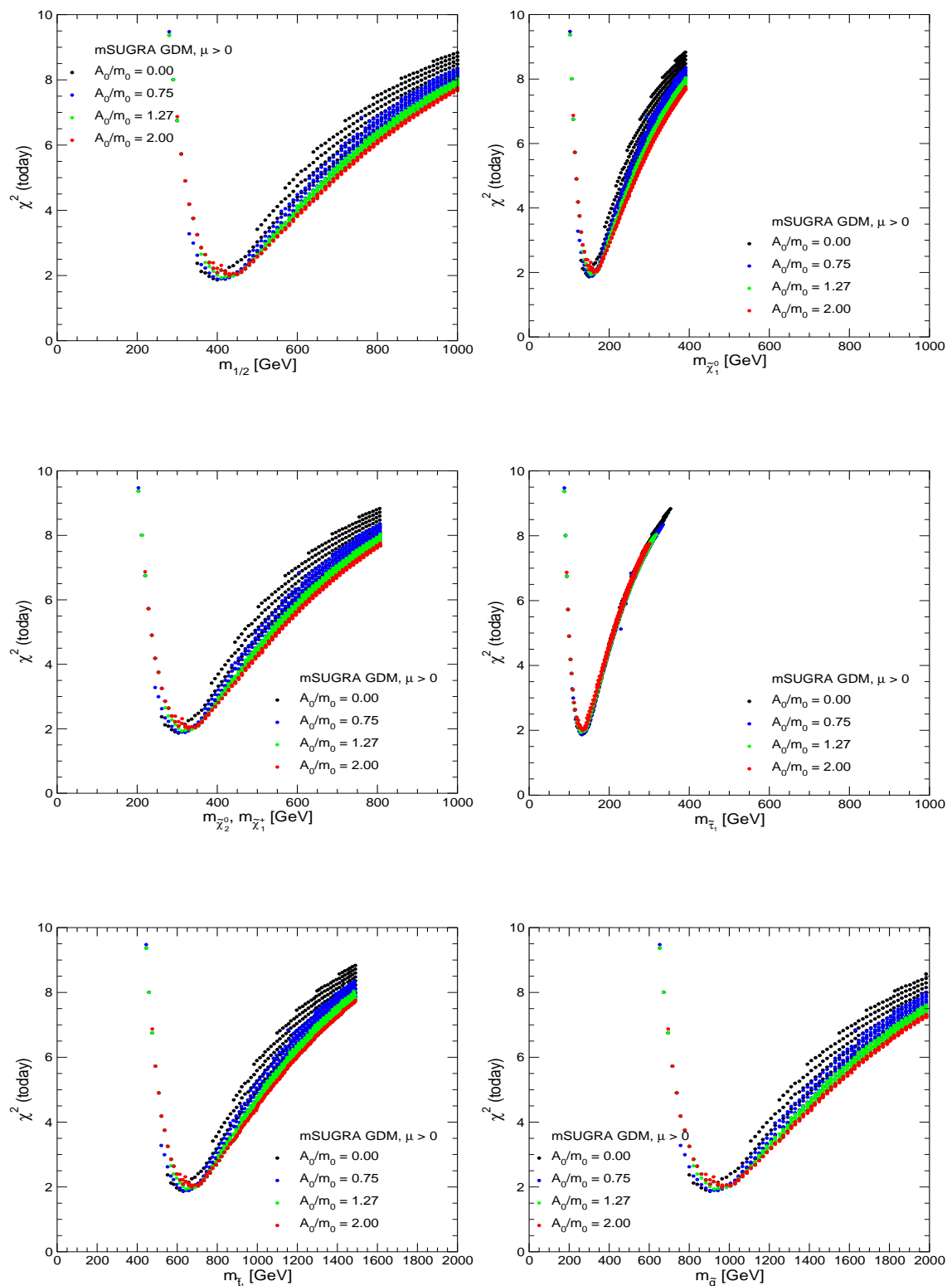

Figure 1. The dependence of the $\chi^{2}$ function on $m_{1 / 2}$ for GDM scenarios with $A_{0} / m_{0}=0,0.75,3-\sqrt{3}$ and 2 , scanning the regions where the lighter stau $\tilde{\tau}_{1}$ is the NLSP, shown as a function of (a) $m_{1 / 2}$, (b) $m_{\tilde{\chi}_{1}^{0}}$, (c) $m_{\tilde{\chi}_{2}^{0}}$ and $m_{\tilde{\chi}_{1}^{ \pm}}$, (d) $m_{\tilde{\tau}_{1}}$, (e) $m_{\tilde{t}_{1}}$, and (f) $m_{\tilde{g}}$. 
the gravitino and a $\tau$, and is metastable with a lifetime that may be measured in hours, days or weeks.

One feature of the class of GDM scenarios discussed here is that the required value of $\tan \beta$ increases with $m_{1 / 2}$. Therefore, the preference for relatively small $m_{1 / 2}$ discussed above maps into an analogous preference for moderate $\tan \beta$, see Ref. [2]. It can be shown that, at the $95 \%$ confidence level

$$
300 \mathrm{GeV} \lesssim \mathrm{m}_{1 / 2} \lesssim 800 \mathrm{GeV}, \quad 15 \lesssim \tan \beta \lesssim 27
$$

in this mSUGRA class of GDM models.

\section{Acknowledgements}

I thank J. Ellis, K.A Olive and G. Weiglein with whom I derived the results presented here.

\section{References}

[1] J. Ellis, S. Heinemeyer, K. Olive, G. Weiglein, JHEP 0502 (2005) 013.

[2] J. Ellis, S. Heinemeyer, K. Olive, G. Weiglein, JHEP 0605 (2006) 005.

[3] J. Ellis, K. Olive, Y. Santoso, V. Spanos, Phys. Rev. D 69 (2004) 095004; B. Allanach, C. Lester, Phys. Rev. D 73 (2006) 015013; B. Allanach, Phys. Lett. 635 (2006) 123; R. de Austri, R. Trotta, L. Roszkowski, JHEP 0605 (2006) 002; hep-ph/0611173; B. Allanach, C. Lester and A.M. Weber, hep-ph/0609295.

[4] J. Ellis, J. Kim, D. Nanopoulos, Phys. Lett. B 145 (1984) 181; T. Moroi, H. Murayama, M. Yamaguchi, Phys. Lett. 303 (1993) 289.

[5] J. Feng, A. Rajaraman and F. Takayama, Phys. Rev. Lett. 91 (2003) 011302; Phys. Rev. D 68 (2003) 063504; J. Feng, S. Su and F. Takayama, Phys. Rev. D 70 (2004) 063514; Phys. Rev. 70 (2004) 075019.

[6] J. Ellis, K. Olive, Y. Santoso, V. Spanos, Phys. Lett. B 588 (2004) 7.

[7] S. Heinemeyer, W. Hollik, G. Weiglein, Phys. Rept. 425 (2006) 265.

[8] C. Bennett et al., Astrophys. J. Suppl. 148 (2003) 1; D. Spergel et al. [WMAP Collaboration], Astrophys. J. Suppl. 148 (2003) 175.

[9] J. Ellis, K. Olive, E. Vangioni, Phys. Lett. B 619 (2005) 30.

[10] CDF Collaboration, D0 Collaboration, Tevatron EWWG, hep-ex/0507091.

[11] J. Ellis, S. Heinemeyer, K. Olive, G. Weiglein, hep-ph/0508169.

[12] LEP Higgs working group, Phys. Lett. B 565 (2003) 61.

[13] For a recent re-evaluation of $M_{W}$ see: S. Heinemeyer, W. Hollik, D. Stöckinger, A.M. Weber, G. Weiglein, JHEP 0608 (2006) 052; hep-ph/0611371;

[14] G. Belanger, F. Boudjema, A. Pukhov, A. Semenov, hep-ph/0405253; C. Degrassi, P. Gambino, G. Giudice, JHEP 0012 (2000) 009; P. Gambino, M. Misiak, Nucl. Phys. B 611 (2001) 338; K. Chetyrkin, M. Misiak, M. Münz, Phys. Lett. B 400, (1997) 206 [Erratum-ibid. B 425 (1998) 414]; G. Hiller, F. Krüger, Phys. Rev. D 69 (2004) 074020; M. Carena, D. Garcia, U. Nierste, C. Wagner, Phys. Lett. B 499 (2001) 141.

[15] T. Moroi, Phys. Rev. D 53 (1996) 6565 [Erratum-ibid. D 56 (1997) 4424]; G. Degrassi, G. Giudice, Phys. Rev. D 58 (1998) 053007; S. Heinemeyer, D. Stöckinger, G. Weiglein, Nucl. Phys. B 690 (2004) 62; Nucl. Phys. B 699 (2004) 103; D. Stöckinger, hep-ph/0609168.

[16] S. Heinemeyer, W. Hollik, G. Weiglein, Comp. Phys. Comm. 1242000 76; Eur. Phys. J. C 9 (1999) 343; G. Degrassi, S. Heinemeyer, W. Hollik, P. Slavich, G. Weiglein, Eur. Phys. J. C 28 (2003) 133; T. Hahn, S. Heinemeyer, W. Hollik, G. Weiglein, hep-ph/0507009; M. Frank, T. Hahn, S. Heinemeyer, W. Hollik, H. Rzehak, G. Weiglein, hep-ph/0611326. T. Hahn, S. Heinemeyer, W. Hollik, H. Rzehak, G. Weiglein, K. Williams, hep-ph/0611373. The code is accessible via www. feynhiggs.de. 